\title{
Alveolar formation is dysregulated by restricted nutrition but not excess sedation in preterm lambs managed by noninvasive support
}

\author{
Lisa A. Joss-Moore ${ }^{1,2}$, Synneva J. Hagen-Lillevik², Calan Yost ${ }^{1}$, Jennifer Jewell ${ }^{1}$, Robert D. Wilkinson ${ }^{1}$, Sydney Bowen ${ }^{1}$, \\ Mar Janna Dahl ${ }^{1}$, Li Dong ${ }^{1}$, Zhengming Wang ${ }^{1}$, Angela P. Presson ${ }^{1,3}$, Chong Zhang ${ }^{3}$, Donald M. Null ${ }^{1,4}$, Bradley A. Yoder ${ }^{1}$ and \\ Kurt H. Albertine ${ }^{1}$
}

\begin{abstract}
BACKGROUND: Preterm birth and respiratory support with invasive mechanical ventilation frequently leads to bronchopulmonary dysplasia (BPD). A hallmark feature of BPD is alveolar simplification. For our preterm lamb model of BPD, invasive mechanical ventilation is associated with postnatal feeding intolerance (reduced nutrition) and sedation. In contrast, preterm lambs managed by noninvasive support (NIS) have normal alveolar formation, appropriate postnatal nutrition, and require little sedation. We used the latter, positive-outcome group to discriminate the contribution of reduced nutrition vs. sedation on alveolar simplification. We hypothesized that, restricted nutrition, but not sedation with pentobarbital, contributes to impaired indices of alveolar formation in preterm lambs managed by NIS.
\end{abstract}

METHODS: Preterm lambs managed by NIS for 21d were randomized into three groups: NIS control, NIS plus restricted nutrition, and NIS plus excess sedation with pentobarbital. We quantified morphological and biochemical indices of alveolar formation, as well as mesenchymal cell apoptosis and proliferation. RESULTS: Restricted nutrition impaired morphological and biochemical indices of alveolar formation, and reduced mesenchymal cell apoptosis and proliferation. Excess sedation with pentobarbital did not alter these indices, although mesenchymal cell apoptosis was less.

CONCLUSION: Our results demonstrate that restricted nutrition, but not excess sedation, contributes to impaired alveolar formation during the evolution of BPD in chronically ventilated preterm lambs.

$\mathrm{P}$ reterm birth accompanied by respiratory support increases the risk for bronchopulmonary dysplasia (BPD; also called neonatal chronic lung disease) (1-3). Even with advances in respiratory support and neonatal intensive care of preterm human infants, BPD remains a significant pediatric problem, with $\sim 15,000$ new cases each year in the United States (4).
Development of BPD is multi-factorial. Contributing factors include invasive mechanical ventilation with oxygenrich gas $(1,2,5-7)$. Prolonged invasive mechanical ventilation with oxygen-rich gas contributes to the development of BPD by disrupting the developmental processes of alveolar formation and capillary growth, leading to the histopathological characteristic of alveolar simplification $(8,9)$. For the focus of this study, inadequate postnatal nutrition and poor growth are also associated with the evolution of BPD (10-12). Inadequate postnatal nutrition and poor growth of preterm infants occurs secondary to enteral feeding intolerance, clinically indicated fluid restriction, and micro/macro-nutrient deficiencies $(2,3,11,13)$. Inadequate nutrition and poor growth are associated with impaired alveolar formation $(14,15)$.

A secondary factor contributing to inadequate postnatal nutrition and impaired alveolar formation may be sedation. Sedation is commonly used during invasive mechanical ventilation to minimize discomfort and stress $(16,17)$. Postnatal sedation may reduce postnatal nutrition and growth via effects on gastrointestinal motility, absorption, and blood flow (18-21).

Isolating the contribution of inadequate postnatal nutrition from the contribution of postnatal sedation on alveolar formation in chronically ventilated preterm infants is challenging. Our preterm lamb model of evolving BPD provides an opportunity to distinguish between the effect of inadequate postnatal nutrition and postnatal sedation on alveolar formation, using a respiratory support mode that is associated with alveolar formation: noninvasive support (NIS) (9). For NIS, we used nasal pulsatile flow ventilation because preterm lambs supported by this mode for $21 \mathrm{~d}$ have normal respiratory gas exchange and normal alveolar formation $(6,7)$. NIS has the added positive benefit of postnatal feeding tolerance that leads to intake of larger volumes of ewe's milk, and better postnatal growth $(6,7)$. None of these good outcomes happens when invasive mechanical ventilation is used. Another positive outcome when NIS

'Department of Pediatrics, University of Utah, Salt Lake City, Utah; ${ }^{2}$ Department of Nutrition and Integrative Physiology, University of Utah, Salt Lake City, Utah; ${ }^{3}$ Department of Internal Medicine, University of Utah, Salt Lake City, Utah; ${ }^{4}$ Current address: Division of Neonatology, Department of Pediatrics, University of California at Davis, Sacramento, California. Correspondence: Lisa Joss-Moore (lisa.joss-moore@hsc.utah.edu)

Received 11 March 2016; accepted 2 May 2016; advance online publication 10 August 2016. doi:10.1038/pr.2016.143 
is used is less sedation is necessary compared to invasive mechanical ventilation $(6,7)$. Therefore, use of NIS to support preterm lambs provides a unique opportunity to test the hypothesis that inadequate nutrition will have a greater effect on morphological and biochemical indices of alveolar formation than excess sedation.

Three groups of preterm lambs managed by NIS are as follows: NIS control, NIS plus restricted nutrition, and NIS plus excess sedation. The primary finding of our study is that restricted nutrition, but not excess sedation, impairs morphological, physiological, and biochemical indices of alveolar formation in chronically ventilated preterm lambs. Our results demonstrate that inadequate nutrition is independently detrimental to alveolar formation in chronically ventilated preterm lambs.

\section{RESULTS}

\section{Preterm Lamb Characteristics}

Preterm lambs were randomized to one of the following three groups: (i) NIS control group $(n=5)$, (ii) NIS plus restricted nutrition (NIS+RN), and (iii) NIS plus excess sedation (NIS+ES). Birth weights of lambs did not vary significantly across the three groups. Sample size was too small to assess sex-specific effects.

Targeted oxygenation and ventilation were $\mathrm{PaO}_{2}$ 60-90 $\mathrm{mmHg}$ and $\mathrm{PaCO}_{2} 45-60 \mathrm{mmHg}$, respectively. Respiratory support parameters are summarized in Table 1. FiO2, PIP and MAP are not different between the NIS control vs. the NIS+RN and NIS+ES groups. During the third week of life, the preterm lambs' activity precluded keeping the nasal tube in place so

Table 1. Respiratory support parameters with interventions for preterm lambs managed by noninvasive support (NIS) for $21 \mathrm{~d}$ (mean $\pm \mathrm{SD})$

\begin{tabular}{|c|c|c|c|c|}
\hline Parameter & Day of life & NIS & $\mathrm{NIS}+\mathrm{RN}$ & $\mathrm{NIS}+\mathrm{ES}$ \\
\hline \multirow[t]{5}{*}{$\mathrm{FiO}_{2}{ }^{\mathrm{a}}$} & 1 & $0.40 \pm 0.11$ & $0.47 \pm 0.13$ & $0.41 \pm 0.18$ \\
\hline & $2-4$ & $0.33 \pm 0.15$ & $0.33 \pm 0.17$ & $0.40 \pm 0.22$ \\
\hline & $6-8$ & $0.30 \pm 0.10$ & $0.31 \pm 0.11$ & $0.30 \pm 0.09$ \\
\hline & $13-15$ & $0.28 \pm 0.03$ & $0.35 \pm 0.12$ & $0.25 \pm 0.06$ \\
\hline & $19-21^{\mathrm{a}}$ & - & - & - \\
\hline $\begin{array}{l}\text { Blow-by flow of } \\
\text { oxygen }^{b}(1 / \mathrm{min})\end{array}$ & $19-21$ & None & $8 \pm 2 *$ & $1 \pm 1$ \\
\hline \multirow{5}{*}{$\begin{array}{l}\text { Peak Inspiratory } \\
\text { Pressure }\left(\mathrm{cmH}_{2} \mathrm{O}\right)^{\text {a }}\end{array}$} & 1 & $16 \pm 2$ & $15 \pm 3$ & $16 \pm 5$ \\
\hline & $2-4$ & $7 \pm 2$ & $7 \pm 6$ & $6 \pm 4$ \\
\hline & $6-8$ & $5 \pm 2$ & $6 \pm 7$ & $3 \pm 2$ \\
\hline & $13-15$ & $3 \pm 1$ & $2 \pm 2$ & $2 \pm 1$ \\
\hline & $19-21^{\mathrm{a}}$ & - & - & - \\
\hline \multirow{5}{*}{$\begin{array}{l}\text { Mean Airway } \\
\text { Pressure }\left(\mathrm{cmH}_{2} \mathrm{O}\right)^{\text {a }}\end{array}$} & 1 & $9 \pm 2$ & $8 \pm 2$ & $8 \pm 2$ \\
\hline & $2-4$ & $5 \pm 1$ & $5 \pm 4$ & $2 \pm 1$ \\
\hline & $6-8$ & $4 \pm 1$ & $4 \pm 4$ & $4 \pm 2$ \\
\hline & $13-15$ & $2 \pm 1$ & $1 \pm 1$ & $2 \pm 1$ \\
\hline & $19-21^{\mathrm{a}}$ & - & - & - \\
\hline
\end{tabular}

${ }^{*} P \leq 0.05$ compared to NIS and NIS+ES a measured at the ventilator. ${ }^{\text {}}$ No nasal tube. blow-by $\mathrm{O}_{2}$ was provided as needed to maintain $\mathrm{O}_{2}$ saturation between 88 and $92 \%$. Flow of blow-by $\mathrm{O}_{2}$ was lower for the NIS control and NIS+ES groups compared to the NIS+RN. Weight gain of the lambs and nutritional parameters were assessed on the final day of study, day of life (DOL) 20, and over two consecutive phases of the study, phase I and phase II.

At DOL20, weight gain was significantly less in the NIS+RN group than the NIS control group (Table 2). Consistent with decreased weight gain at DOL 20, NIS+RN lambs received fewer total calories, fluid, protein and fat than NIS control lambs. At DOL 20 NIS+ES lambs weight gain, total calories, fluid, protein and fat was not different from NIS control lambs (Table 2). During phase I, there were no daily differences in NIS+RN lamb weight gain, calories, fluid, protein, or fat compared to NIS control lambs. In phase II, daily weight gain, calories, fluid, protein, and fat for NIS+RN group was significantly less than NIS control lambs (Table 2). During phase I, there was no difference in daily weight gain between NIS+ES lambs and NIS control lambs. However, during phase I, NIS+ES lambs had greater daily calories, fluid, fat and protein than NIS control lambs. In phase II, daily weight gain was also not different between NIS+ES and NIS control lambs. However, in phase II, daily calories, fluid, protein, and fat was significantly less in NIS+ES lambs than NIS control lambs (Table 2).

\section{Effect of Restricted Nutrition on Lung Outcomes}

Histologically, terminal respiratory units appear simplified in the NIS+RN group compared to the NIS control group (Figure 1). For the NIS+RN group, the lung parenchyma had thick airspace walls and few secondary septa. Secondary septa that were present were short and thick. These simplified features were different from the lung parenchyma for the NIS control group. That group's lung parenchyma had thin airspace walls and many secondary septa that were long and thin. Morphometry and stereology substantiated these impressions (Figure 1). The quantitative histological results showed that structural indices of alveolar formation were significantly different between the NIS+RN and NIS control groups (Figure 1). The NIS+RN group had significantly lower radial alveolar count and volume density of alveolar secondary septa compared to the NIS control group. Conversely, the NIS+RN group had significantly thicker distal airspace walls compared to the NIS control group.

For efficient respiratory gas exchange, the lung's parenchyma must become thin. Thinning occurs through gradual increased apoptosis, and decreased proliferation, of mesenchymal cells in the walls of the distal airspaces (saccules) (6). Therefore, we quantified biochemical markers of apoptosis and proliferation, and localized both protein markers (Figure 2). Relative abundance of the pro-apoptotic protein, cleaved caspase-3, was significantly lower for the NIS+RN group compared to the NIS control group. PCNA protein abundance, a marker of cellular proliferation, also was significantly lower for the NIS+RN group compared to the NIS control group. Immunohistochemistry showed that fewer mesenchymalcell nuclei were immunostained brown for cleaved caspase 3 
Table 2. Linear mixed model estimates and $95 \%$ confidence intervals for weight gain and nutrition parameters for preterm lambs managed by noninvasive support (NIS) for $21 \mathrm{~d}$

\begin{tabular}{|c|c|c|c|c|}
\hline & & \multirow[b]{2}{*}{ NIS $(n=5)$} & \multicolumn{2}{|c|}{ Difference from NIS } \\
\hline & & & $\mathrm{NIS}+\mathrm{RN}(n=4)$ & $\mathrm{NIS}+\mathrm{ES}(n=4)$ \\
\hline Weight gain at DOL20 (kg) & & $1.46(0.97 \sim 1.94)$ & $-1.24(-1.97 \sim-0.51)^{*}$ & $0.37(-0.36 \sim 1.10)$ \\
\hline Daily weight gain (kg) & Phase II ${ }^{\mathrm{b}}$ & $0.19(0.15 \sim 0.23)$ & $-0.19(-0.25 \sim-0.13)^{*}$ & $\leq 0.00(-0.06 \sim 0.06)$ \\
\hline Total calories on DOL20 (kcal/kg) & & $283(249 \sim 318)$ & $-211(-262 \sim-159)^{*}$ & $-41(-93 \sim 10)$ \\
\hline Daily change in calories (kcal/kg) & Phase II & $15(11 \sim 20)$ & $-29(-36 \sim-23)^{*}$ & $-15(-21 \sim-8)^{*}$ \\
\hline Total fluid on DOL20 (ml/kg) & & $283(245 \sim 320)$ & $-136(-193 \sim-79)^{*}$ & $-31(-88 \sim 26)$ \\
\hline \multirow[t]{2}{*}{ Daily change in fluid intake (ml/kg) } & Phase I & $4(1 \sim 7)$ & $1(-4 \sim 5)$ & $6(1 \sim 10)^{*}$ \\
\hline & Phase II & $6(1 \sim 10)$ & $-18(-25 \sim-12)^{*}$ & $-8(-14 \sim-1)^{*}$ \\
\hline Protein on DOL20 (g/kg) & & $15.6(13.7 \sim 17.6)$ & $-12.2(-15.2 \sim-9.3)^{*}$ & $-2.1(-5.1 \sim 0.8)$ \\
\hline \multirow[t]{2}{*}{ Daily change in fat intake $(\mathrm{g} / \mathrm{kg})$} & Phase I & $0.7(0.6 \sim 0.9)$ & $0.1(-0.2 \sim 0.4)$ & $0.5(0.2 \sim 0.7)^{*}$ \\
\hline & Phase II & $1.0(0.7 \sim 1.3)$ & $-2.0(-2.4 \sim-1.5)^{*}$ & $-0.9(-1.4 \sim-0.5)^{*}$ \\
\hline
\end{tabular}

aphase I is postnatal DOL 5-12 for weight gain, and DOL 1-12 for all other parameters. ${ }^{\text {PPhase }}$ II is postnatal DOL $13-20 .{ }^{*} \leq 0.05$ compared to NIS.

DOL, day of life; NIS, noninvasive support; RN, restricted nutrition; ES, excess sedation.

protein and PCNA protein for the NIS+RN group compared to the NIS control group.

\section{Effect of Excess Sedation on Lung Outcomes}

Histologically, terminal respiratory units appeared similar between the NIS+ES and NIS control groups (Figure 3). Quantitative morphometric and stereologic measurements showed that the three structural indices of alveolar formation were similar between NIS+ES and NIS control groups.

Relative abundance of cleaved caspase- 3 protein was significantly lower for the NIS+ES group compared to the NIS control group (Figure 4). However, relative abundance of PCNA protein was not different between the NIS+ES and NIS control groups. Immunohistochemistry showed that fewer mesenchymal-cell nuclei were immunostained brown for cleaved caspase 3 protein. The immunostain pattern for PCNA protein was similar between the NIS+ES and NIS control groups (Figure 4).

\section{DISCUSSION}

Our study provides the new insight that restricted nutrition for $21 \mathrm{~d}$ in preterm lambs managed by NIS has a greater effect on alveolar formation than excess sedation. Preterm lambs managed by NIS that received restricted nutrition have impaired morphological, physiological, and biochemical indices of alveolar formation, as well as reduced mesenchymal cell apoptosis and proliferation. Preterm lambs managed by NIS that received excess sedation have normal morphological, physiological, and biochemical indices of alveolar formation, although with less mesenchymal cell apoptosis. Therefore, our study demonstrates the importance of adequate nutrition compared to sedation for appropriate alveolar formation in chronically ventilated preterm lambs.

The novelty of our study is that we used preterm lambs managed by NIS for $21 \mathrm{~d}$ to discriminate between the impacts of limited nutrition vs. sedation. We focused on NIS-managed preterm lambs because they tolerate enteral feedings and grow, and they require little sedation. In addition, NIS-managed preterm lambs have acceptable respiratory gas exchange that requires less supplemental oxygen, lower peak inspiratory pressure, and lower mean airway pressure than that are required to support preterm lambs by invasive mechanical ventilation $(6,7)$. NIS-managed preterm lambs have appropriate alveolar formation, whereas, preterm lambs supported by invasive mechanical ventilation have alveolar simplification $(6,7)$. Therefore, we used the good outcomes of NIS-managed preterm lambs as the context for asking if alveolar simplification occurs because of limiting enteral feedings (restricted nutrition (RN)) or providing sedation (excess sedation (ES)). Because we kept respiratory gas exchange within acceptable ranges for $\mathrm{PaO}_{2}, \mathrm{PaCO}_{2}, \mathrm{pH}$, and $\mathrm{O}_{2}$ saturation for all three groups of NIS-managed preterm lambs, the principal independent variables for our study are restricted nutrition and excess sedation.

The restricted nutrition protocol that we used is based on enteral intake by historical preterm lambs supported by invasive mechanical ventilation for $21 \mathrm{~d}$ who developed feeding intolerance during the third week of life $(6,7)$. Our restricted nutrition protocol provided the NIS+RN lambs with matched volumes $(\mathrm{ml} / \mathrm{kg} / \mathrm{d})$ of ewe milk tolerated daily by the historical preterm lambs. We used linear mixed effects model analysis to compare NIS, NIS+RN, and NIS+ES groups, fitting separate lines to the 

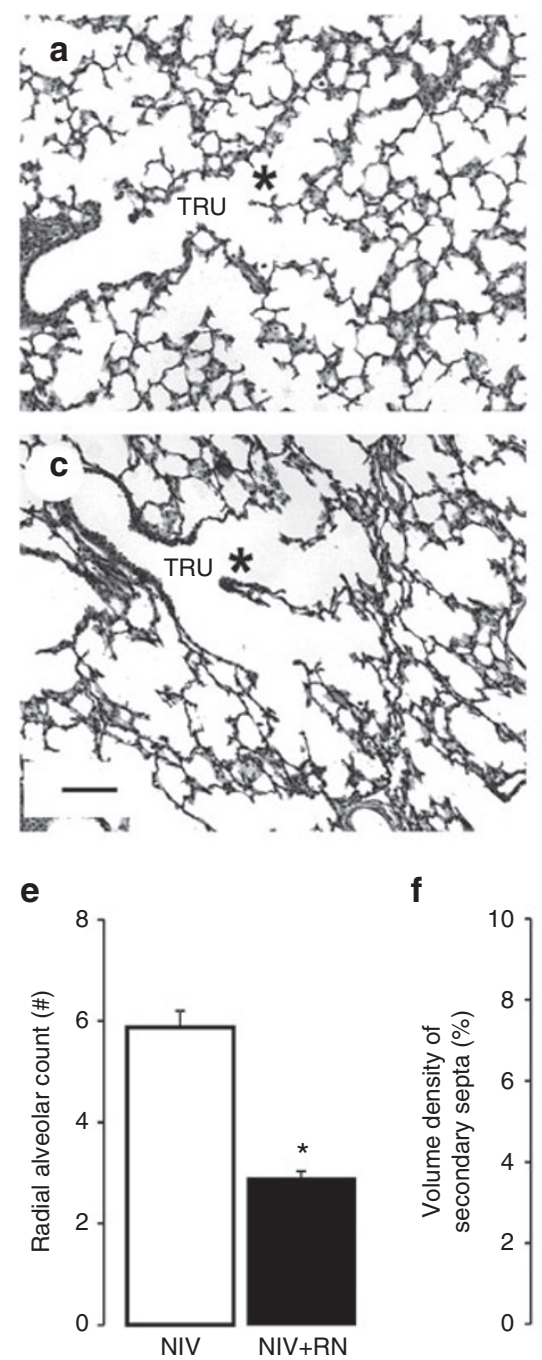

f



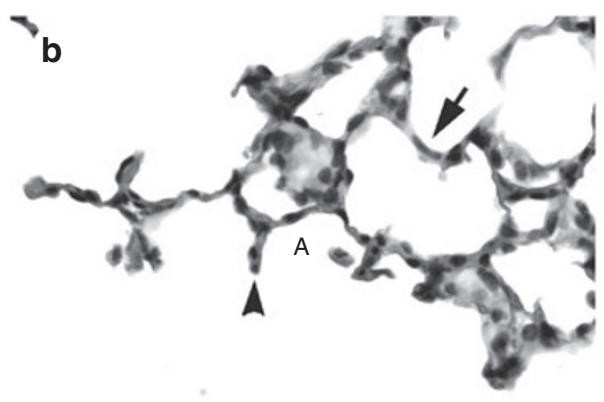

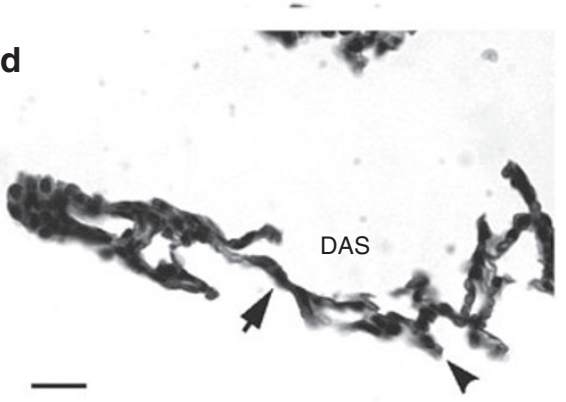

g

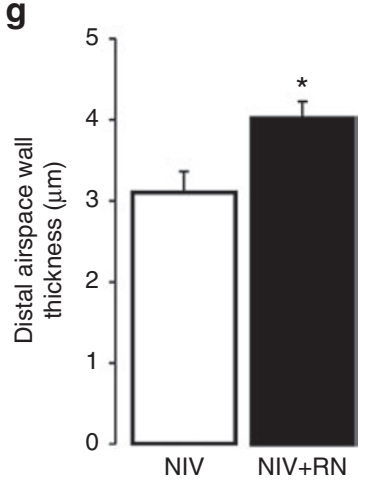

Figure 1. Histologic appearance and morphometric quantification of lung structure in preterm lambs supported by NIS, with or without restricted nutrition (RN). (a and b) NIS control lung. (c and $\mathbf{d}$ ) NIS+RN lung. A, alveolus; DAS, distal airspace; TRU, terminal respiratory unit. * identifies the enlarged region in panels $\mathbf{b}$ and $\mathbf{d}$. Lung parenchyma of the NIS control group has thinner distal airspace walls (arrow), and more and thinner alveolar secondary septa (arrowhead) compared to corresponding NIS+RN group (panels a and c, the scale bar is $20 \mu \mathrm{m}$; panels $\mathbf{b}$ and $\mathbf{d}$, the scare bar is $100 \mu \mathrm{m}$ ). Images are representative of the morphometric results shown in panels e, $\mathbf{f}$, and $\mathbf{g}$. Morphometric results are shown as mean \pm SD for $n=4 / g r o u p$. White fill indicates NIS control; black fill indicates NIS+RN. Radial alveolar count (panel e) and volume density of secondary septa (panel f) are significantly greater in the NIS control group compared to the NIS+RN group. Distal airspace wall thickness (panel $\mathbf{g}$ ) is significantly less in the NIS control group compared to the NIS+RN group. *Different from the NIS control group by Mann-Whitney U-test $(P<0.05)$.

first and second phases of life. This was the simplest model that best described the weight and nutrition data, and was made possible by the presence of 1 inflection point in the data between day of life 12 and 13. The NIS + RN lambs received similar enteral feeding volumes NIS control lambs during phase I of life (days 1-12). During phase II of life (days 13-20), NIS+RN lambs received less daily enteral feeding volume, and therefore less daily total calories, than NIS control lambs. Consistent with reduced caloric intake, weight gain was less during phase II for the of NIS+RN lambs compared to the NIS control lambs. The decline in daily enteral volume, total calories, and weight gain in NIS+RN lambs was accompanied by alveolar simplification compared to the NIS control group. The outcomes for the NIS+RN group highlight the importance of sustained adequate nutrition during the entire period of respiratory support (22).
Our findings add to a body of work that examines the link between postnatal nutrition, alveolar structural development and BPD. In adult mice, restricted nutrition leads to loss of alveoli, while restoration of nutrition leads to regeneration of alveoli $(14,23)$. In rat studies, direct nutritional restriction in the pre- and postnatal periods, as well as uteroplacental insufficiency, result in impaired alveolar formation $(15,24)$. Our study confirms those findings, in a clinically relevant model by showing that restricted nutrition in the immature lung of chronically ventilated preterm lambs leads to alveolar simplification that involves less apoptosis and proliferation of mesenchymal cells in the walls of distal airspaces. Our recent studies showed that balance between apoptosis and proliferation of mesenchymal cells is sensitive to the mode of respiratory support in chronically ventilated preterm lambs $(6,7)$. The balance is shifted in 
a

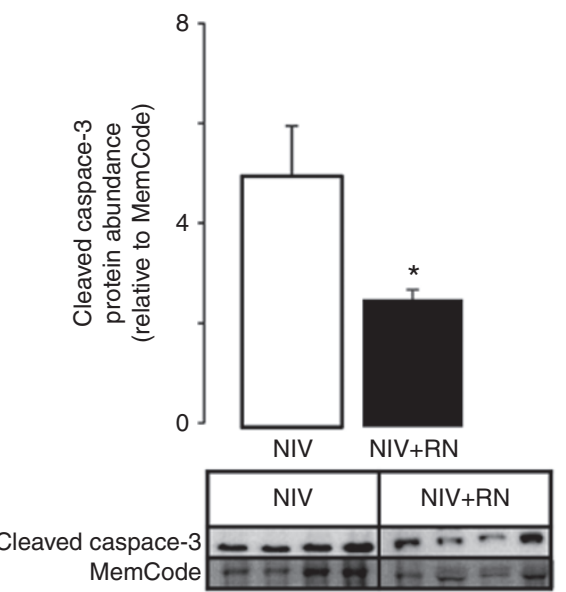

b

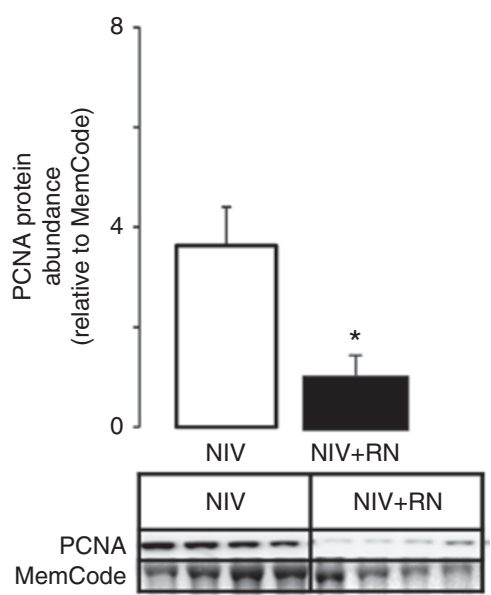

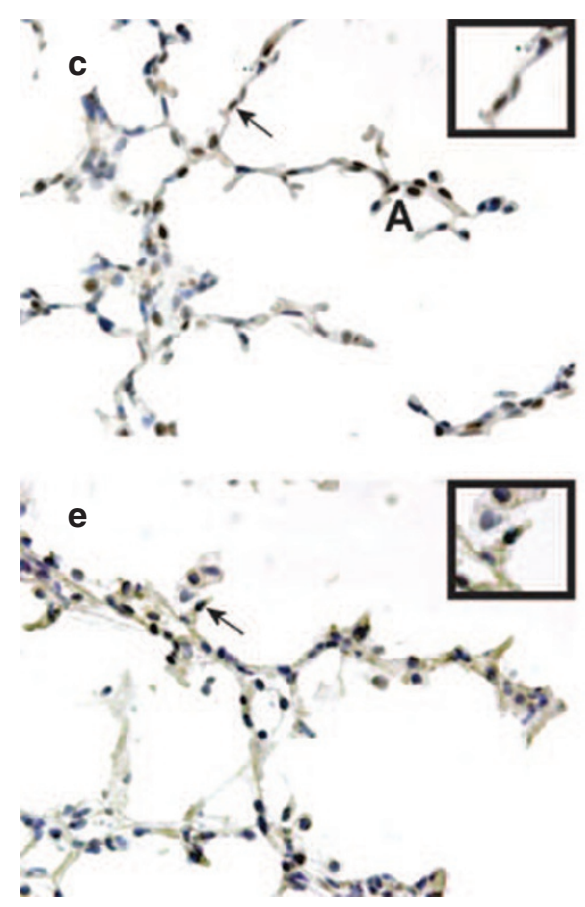

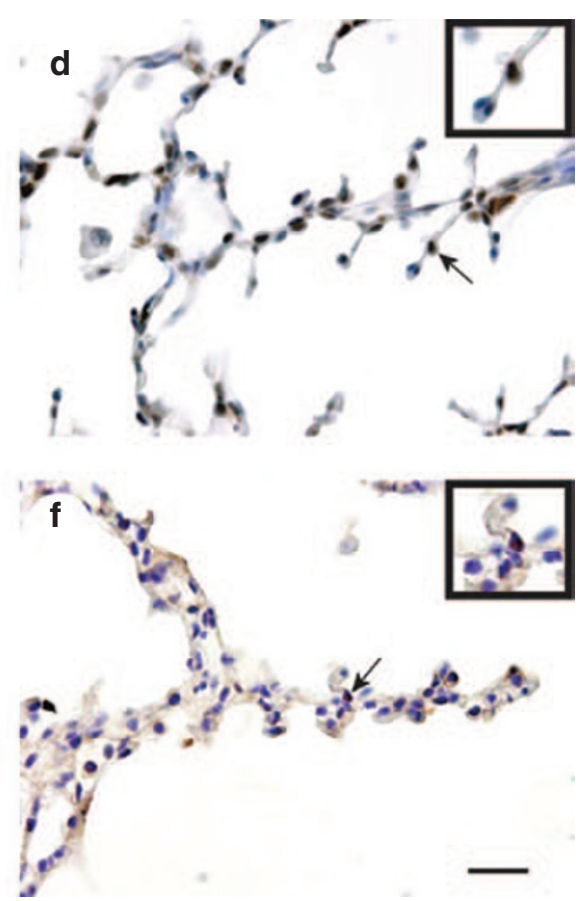

Figure 2. Quantification of cleaved caspase-3 and PCNA protein abundance in preterm lambs supported by NIS, with or without restricted nutrition (RN). White fill indicates NIS control, black fill indicates NIS+RN. Results are shown as mean \pm SD for $n=4$ /group. (a) Cleaved caspase-3 protein abundance. (b) PCNA protein abundance. Cleaved caspase-3 and PCNA protein abundance are significantly lower in lungs of NIS+RN group compared to NIS control group (*different from the NIS group by Mann-Whitney U-test, $P<0.05$ ). (c and d) NIS immunohistochemistry of (c) cleaved caspase 3 and (d) PCNA. (e and f) NIS+RN immunohistochemistry of (e) cleaved caspase 3 and (f) PCNA. Immunohistochemistry shows that fewer mesenchymal-cell nuclei are immunostained brown for cleaved caspase 3 protein in NIS+RN and PCNA protein for the NIS+RN group compared to the NIS control group (scale bar is $100 \mu \mathrm{m})$. Insets are enlargements of the region identified by the arrow in each panel.

favor of apoptosis during NIS respiratory management, whereas the balance is shifted in favor of proliferation during invasive mechanical ventilation. Based on the results of the present study, we propose that restricted nutrition disrupts the balance, too, but differently, in that restricted nutrition leads to static turnover of mesenchymal cells in the walls of distal airspaces (Figure 2). Static turnover may contribute to the persistently thick walls of the distal airspaces (Figure 1). An implication of our results is that alveolar simplification in the lung of preterm infants supported by invasive mechanical ventilation may be the combined manifestation of invasive mechanical ventilation and inadequate nutrition due to feeding intolerance.
Despite aggressive nutritional management, optimal nutritional delivery to preterm infants can be difficult because of physiologic and management limitations $(1,2,25)$. Postnatal growth restriction also occurs in a significant number of preterm infants, and is frequently more severe with earlier preterm birth or greater disease severity (25-28). To facilitate adequate nutrition to preterm infants, parenteral nutrition is used during early days of life. Despite the advantage of parental nutrition for early growth and neurodevelopment (10), the amount of enteral nutrition appears to influence the development of BPD. For example, among preterm infants receiving combined enteral and parenteral nutrition, infants who developed BPD 

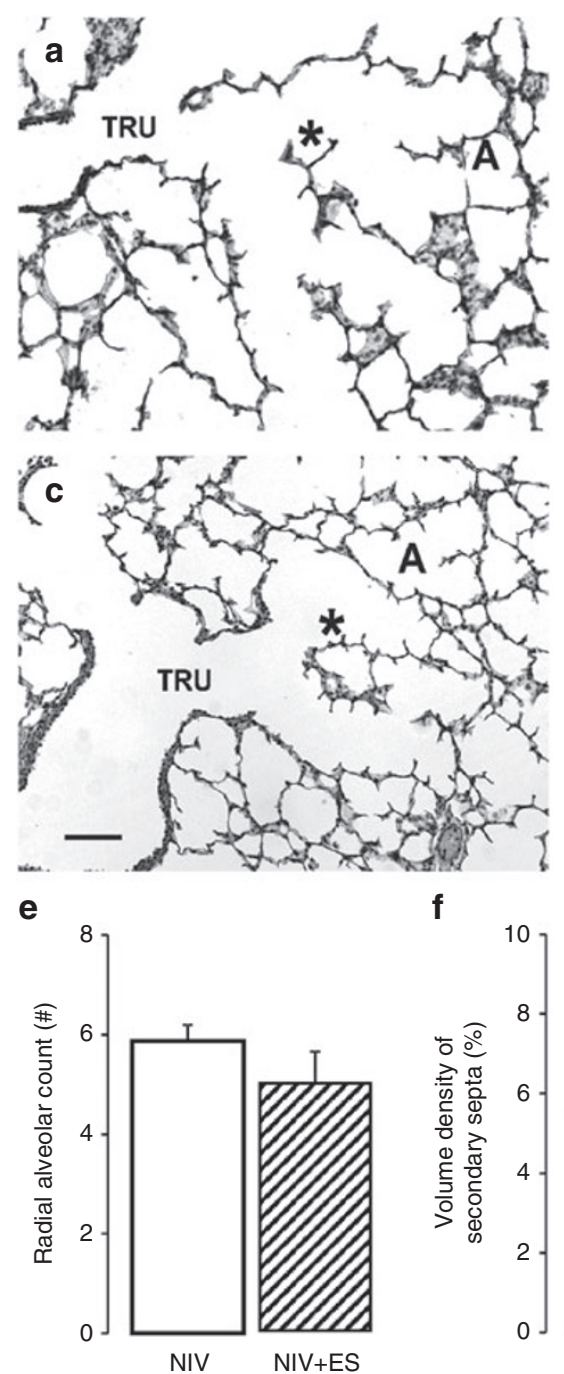
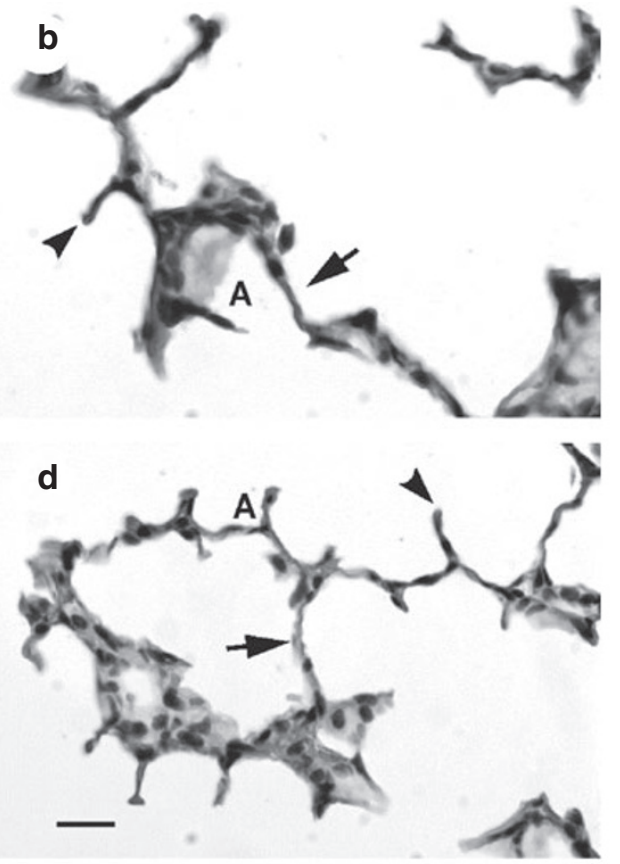

g

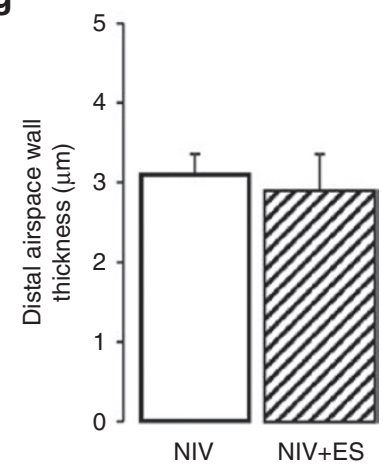

Figure 3. Histologic appearance and morphometric quantification of lung structure in preterm lambs supported by NIS, with or without excess sedation (ES). (a and b) NIS control lung. (c and d) NIS+ES lung. A, alveolus; DAS, distal airspace; TRU, terminal respiratory unit. * identifies the enlarged region in panels $\mathbf{b}$ and $\mathbf{d}$. Lung parenchyma of the NIS control group is similar to the corresponding NIS+ES group (panels a and c, the scale bar is $20 \mu \mathrm{m}$; panels $\mathbf{b}$ and $\mathbf{d}$, the scale bar is $100 \mu \mathrm{m}$ ). Images are representative of the morphometric results shown in panels $\mathbf{e}, \mathbf{f}$, and $\mathbf{g}$. Morphometric results are shown as mean \pm SD. White fill indicates NIS control, hatched fill indicates NIS+ES. Radial alveolar count (panel e), volume density of secondary septa (panel f), and distal airspace wall thickness (panel $\mathbf{g}$ ) is not different between NIS control group and NIS+ES group.

received less enteral feeding than those who did not develop $\mathrm{BPD}$, despite both groups receiving the same total fluid volume, calories, and grams of macronutrient (29). We did not use parenteral nutrition because our goal was to assess the effect of enteral nutrition on alveolar formation. Whether that consequence is avoidable by parenteral nutrition during that critical period of early postnatal development remains to be determined.

Sedation of preterm infants receiving invasive mechanical ventilation may affect alveolar formation directly, by altering lung cell-signaling, or indirectly by prolonging mechanical ventilation, or disrupting nutritional intake and/or absorption $(18,19,30-32)$. In our study, preterm lambs in the NIS+ES group tolerated sheep colostrum and milk. During phase I of life, NIS+ES lambs consumed more milk than the NIS control group, for reasons that are not clear. However, the NIS+ES lambs gained the same amount of weight as the NIS control group during phase I and phase II of life. Increased milk intake and sustained weight gain suggest that gastrointestinal function was not impaired by the dosage of pentobarbital given to the NIS+ES group. Because alveolar formation occurred in the NIS+ES lambs to the same extent as the NIS control lambs, we conclude that sedation, as we applied it, does not lead to alveolar simplification.

A limitation of our study is that parenteral nutrition was not provided (22). New studies are examining the impact of isocaloric nutrition provided enterally and parenterally. A limitation for translation may be use of pentobarbital in our studies. We use pentobarbital because it provides stable, continuous sedation of the lambs for these long, difficult studies. However, pentobarbital is rarely used for preterm infants, and thus the lack of effect on alveolar formation in preterm lambs may not 
a
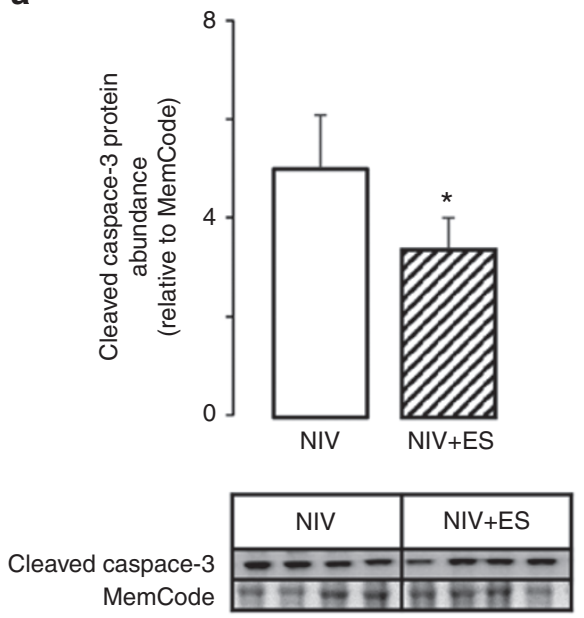

b

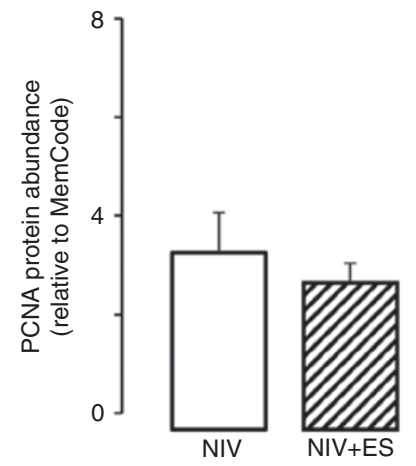

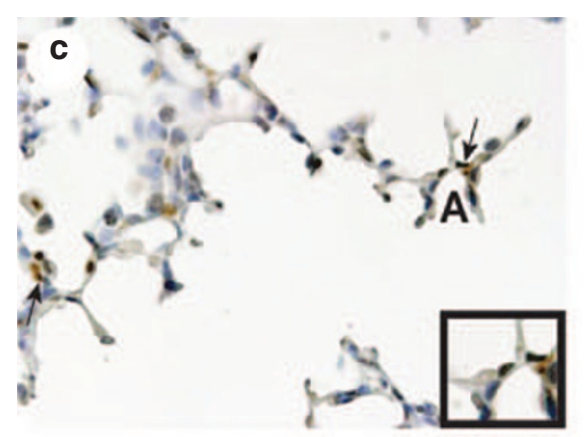
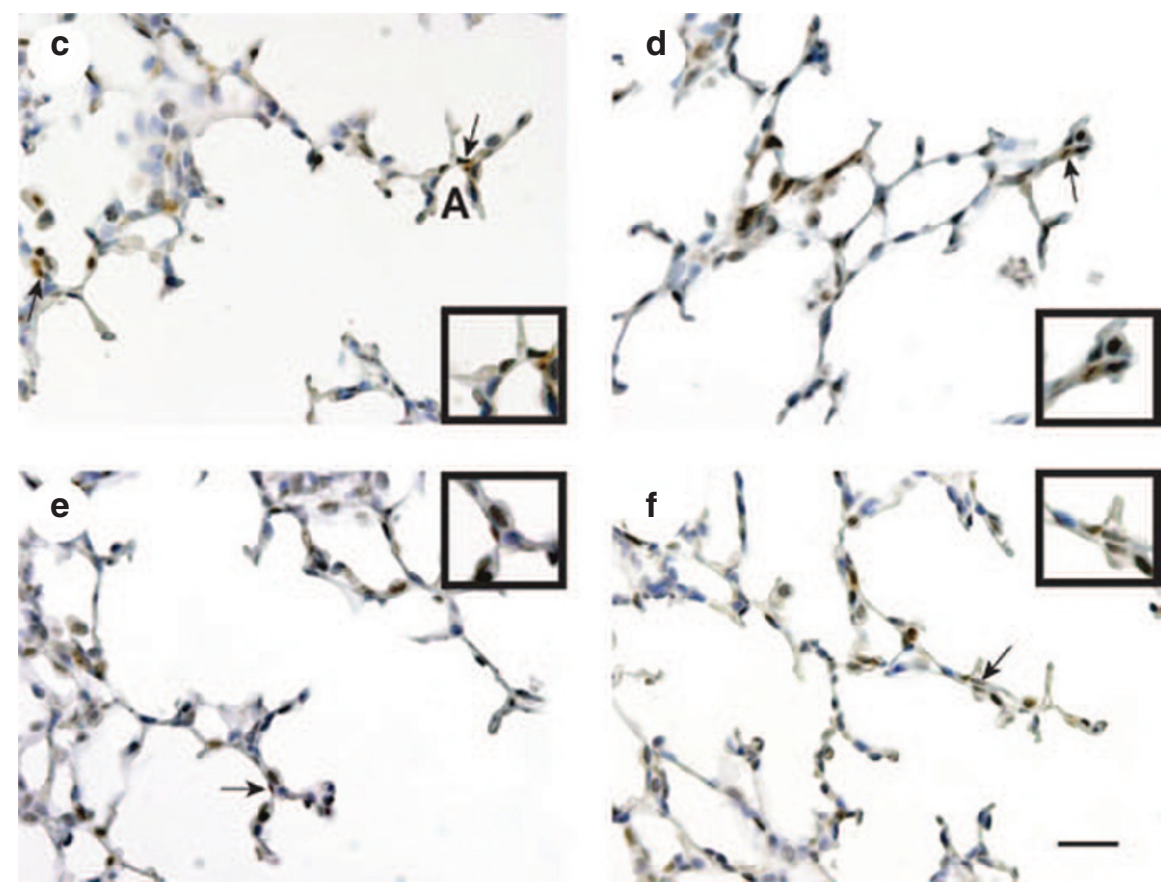

Figure 4. Quantification of cleaved caspase-3 and PCNA protein abundance in preterm lambs supported by NIS, with or without excess sedation (ES). White fill indicates NIS control, hatched fill indicates NIS+ES. Results are shown as mean \pm SD for $n=4 /$ group. (a) Cleaved caspase-3 protein abundance. (b) PCNA protein abundance. Cleaved caspase-3 abundance is significantly lower in lungs of NIS+ES group compared to NIS control group $\left({ }^{*}\right.$ different from the NIS group by Mann-Whitney $U$-test, $P<0.05$ ). (c and d) NIS immunohistochemistry of (c) cleaved caspase 3 and (d) PCNA. (e and f) NIS+ES immunohistochemistry of (e) cleaved caspase 3 and (f) PCNA. Immunohistochemistry showed that fewer mesenchymal-cell nuclei were immunostained brown for cleaved caspase 3 protein. The immunostain pattern for PCNA protein was similar (scale bar is $100 \mu \mathrm{m}$ ). Insets are enlargements of the region identified by the arrow in each panel.

be generalizable to sedatives that are used for preterm infants. The dosage of pentobarbital given to preterm lambs in the NIS+ES group was at the low-end of the dose range used to sedate the historical lambs that were supported by IMV. We used the low-end of the dosage range to avoid interfering with effective spontaneous breathing by the NIS+ES group of lambs. We suggest that the dosage of sedation given to the NIS+ES group of preterm lambs did not contribute to impaired alveolar formation in preterm lambs, lending further support to the major finding of our study, which is that reduced nutrition decreases alveolar formation. Sample size for this study is four to five preterm lambs/group. This sample size reflects balance between sample size requirements for statistical tests vs. animal welfare expectations to use the minimum number of lambs. Consequently, sample size for our preterm lamb studies is small compared to studies of human preterm neonates. The small sample size may make type II statistical error a possibility. However, our cumulative experience is that when sample size is at least four and the data a normally distributed, statistical significance is reached at sample size of $4(6,7)$.

Molecular mechanisms by which restricted nutrition may alter lung growth and alveolar formation remains to be 
identified. One potential mechanism of altered alveolar formation in NIS+RN lambs is oxidant injury. Several studies in rats demonstrate an additive effect of undernutrition and hyperoxic lung injury (33). Another potential mechanism may involve specific bioactive nutrients important in alveolar formation and the development of BPD (3). Of particular interest to our group are essential fatty acids. Preterm infants who develop BPD have alterations in circulating essential fatty acid profiles, including decreased docoshexanoic acid (DHA) (34). Studies from our laboratory show that reduced DHA is associated with impaired molecular signaling and alveolar formation in the lung of intrauterine growth-restricted rat pups (35). Importantly, DHA supplementation restored molecular signaling and alveolar formation (35). We are assessing DHA levels in plasma and lung of chronically ventilated preterm lambs.

In conclusion, our study demonstrates that restricted nutrition, rather than excess sedation, reduces morphological, physiological, and biochemical indices of alveolar formation in chronically ventilated preterm lambs. Our results highlight the independent detrimental effect of inadequate nutrition on alveolar formation in chronically ventilated preterm lambs. Current studies are evaluating the contribution of overall calories, and the contribution of specific macronutrient activation of molecular signaling pathways that participate in alveolar formation.

\section{METHODS}

Surgical Preparation and Management of Preterm Lambs

The protocol for using chronically ventilated preterm lambs was in accordance with the American Physiological Society/US National Institutes of Health guidelines for humane use of animals for research. The protocol was prospectively approved by the Institutional Animal Care and Use Committee at the University of Utah, Health Sciences Center.

The methods for chronically ventilating preterm lambs are reported $(6,7,36,37)$. All lambs were initially intubated and supported by invasive $\mathrm{MV}$ for $\sim 3 \mathrm{~h}$. Initial ventilator settings were: respiratory rate 60 breaths/min, inspiratory time $0.3 \mathrm{~s}$, PEEP $7 \mathrm{cmH}_{2} \mathrm{O}$, flow $8 \mathrm{l} / \mathrm{min}$ of $50 \% \mathrm{O}_{2}$, and expiratory tidal volume $5-7 \mathrm{ml} / \mathrm{kg}$. Lambs were given an intravenous loading dose of caffeine citrate $(15 \mathrm{mg} / \mathrm{kg}$, given over $2 \mathrm{~h}$, Mead Johnson \& Company, Evansville, IN) within $30 \mathrm{~min}$ of delivery, and maintenance doses were $5 \mathrm{mg} / \mathrm{kg} / \mathrm{d}$. Vascular pressures and heart rate were recorded continuously (model V6400; SurgiVet, Waukesha, WI). Intravenous dextrose was given to maintain plasma glucose between 60 and $90 \mathrm{mg} / \mathrm{dl}$. Arterial partial pressure of oxygen $\left(\mathrm{PaO}_{2}\right)$ was targeted between 70 and $90 \mathrm{mmHg}$ by adjusting fractional inspired $\mathrm{O}_{2}\left(\mathrm{FiO}_{2}\right)$. Oxygenation saturation by pulse oximetry (SurgiVet, Smiths Medical, Dublin, OH) was targeted for $88-92 \%$. Arterial partial pressure of carbon dioxide $\left(\mathrm{PaCO}_{2}\right)$ was targeted between 40 and $60 \mathrm{mmHg}$, and $\mathrm{pH}$ between 7.25 and 7.45 , by adjusting peak inspiratory pressure. Arterial blood gases, $\mathrm{pH}$, electrolytes, glucose, and $\mathrm{O}_{2}$ saturation were measured hourly during the first days of life.

All lambs were weaned to NIS, within $\sim 3 \mathrm{~h}$ of life, and were maintained on NIS for $21 \mathrm{~d}(6,7)$. Respiratory support was by pulsatile Flow Ventilation (model VDR4; Percussionaire, Sand Point, ID) through an uncuffed oral/nasal true Murphy tube (3.0 French; $13 \mathrm{~cm}$ length) that was inserted $\sim 5 \mathrm{~cm}$ through a nostril (the nasal cavity is $\sim 10 \mathrm{~cm}$ in length in fetal lambs). Initial ventilator settings were background convective breaths at $\sim 10$ breaths/min, PIP of 20 to $25 \mathrm{cmH}_{2} \mathrm{O}$, PEEP of 5 to $7 \mathrm{cmH}_{2} \mathrm{O}$, and high-frequency rate at 8 to $10 \mathrm{~Hz}$. Target ranges for $\mathrm{PaO}_{2}$ and $\mathrm{PaCO}_{2}$ were maintained by adjusting ventilator settings. Lambs received daily antibiotics for $10 \mathrm{~d}$. Lambs also were given buprenorphine hydrochloride $(5 \mathrm{mcg} / \mathrm{kg}$ every 6 h, Reckitt \& Colman Pharmaceuticals, Richmond, VA).

\section{Study Groups and Management of Preterm Lambs}

To separate the effect of restricted nutrition vs. excess sedation on morphological and biochemical indices of alveolar formation, we randomized NIS-supported preterm lambs to one of three study groups, all of which were supported for $21 \mathrm{~d}$. We took steps to minimize investigator bias during the conduct of this study. We prospectively randomized assignment to a study group before operative delivery of fetal lambs. Sample size was 4 to 5/group for compliance to reduce the number of lambs/group to that necessary to assess statistical differences $(P<0.05)$. Sample size for the NIS control group was 5 because that was the number of control preterm lambs that accrued to reach $n=4$ each for the NIS+RN group and NIS+ES group. One group was supported by NIS with our standard nutritional regimen that leads to postnatal growth (NIS control), another group was supported by NIS with restricted nutrition (NIS+RN), and the third group was supported by NIS with our standard nutritional regimen and excess sedation (NIS+ES). We kept respiratory gas exchange within acceptable ranges for $\mathrm{PaO}_{2}, \mathrm{PaCO}_{2}$, and $\mathrm{pH}$ for all three groups of NIS-supported preterm lambs.

Initial feedings were via an orogastric tube. All lambs received sheep colostrum (Kid \& Lamb Colostrum Replacement, Land O Lakes, Arden Hills, MN) for the first $3 \mathrm{~d}$ of life. The initial volume $(3 \mathrm{ml})$ was increased gradually by $3-5 \mathrm{ml}$ increments, as tolerated, to attain a goal of 60 to $80 \mathrm{kcal} / \mathrm{kg} / \mathrm{d}$. At day of life 4 , colostrum was replaced with mature sheep milk (Sav-A-Lam Milk Products, Chilton, WI). Milk volume was graduated as tolerated by the NIS and NIS+ES groups and lambs fed ad libitum for a target of $150 \mathrm{kcal} / \mathrm{kg} / \mathrm{d}$. For the NIS+RN group, milk restriction was based on the volume of milk $(\mathrm{ml} / \mathrm{kg} / \mathrm{d})$ tolerated by historical preterm lambs managed by invasive mechanical ventilation for $21 \mathrm{~d}(7,36)$. Fluid homeostasis, as indicated by urine output ( $>1$ to $2 \mathrm{ml} / \mathrm{kg} / \mathrm{h}$ ) and mean arterial blood pressure (>45 mmHg) was maintained for all lambs by adjusting total fluid intake (colostrum, milk, saline, dextrose) based on output (withdrawn blood, urine, and stool). None of the lambs required treatment with pressors. Feeding and growth data were collected continuously over each $24 \mathrm{~h}$ period. The studies ended on day of life 21 ; so, daily calculations of intake and output are through day of life 20.

Lambs received pentobarbital (Abbott Laboratories, North Chicago, IL) at 1 to $2 \mathrm{mg} / \mathrm{kg}$, as needed (heart rate $>200$ beats $/ \mathrm{min}$ ). The NIS control and NIS+RN groups received pentobarbital at $0.8 \pm 0.6 \mathrm{mg} /$ $\mathrm{kg} / \mathrm{d}$. The NIS+ES group received pentobarbital at $4.8 \mathrm{mg} / \mathrm{kg} / \mathrm{d}$. This dosage is based on daily dosage $(\mathrm{mg} / \mathrm{kg} / \mathrm{d})$ needed to keep historical preterm lambs supported by invasive mechanical ventilation comfortable and calm $(7,36)$.

\section{Terminal Collection of Tissue}

At necropsy, preterm lambs were given $60 \mathrm{mg} / \mathrm{kg}$ pentobarbital sodium solution intravenously (Beuthanasia solution, Ovation Pharmaceuticals, Deerfield, IL). The lambs were intubated and reconnected to the Percussionaire ventilator to maintain lung inflation when the chest was opened to remove the lungs. Lung tissue preservation (frozen, fixed) was by methods that we reported $(6,7,36,37)$.

\section{Quantification of Alveolar Secondary Septation and Distal Airspace Wall Thickness}

We used morphometry and stereology to quantitatively compare alveolar secondary septation (radial alveolar count and secondary septal volume density) and distal airspace wall thickness, using methods previously reported by our group $(7,36)$. We used unbiased (systematic, uniform, random) sampling of the uniformly insufflated, whole left lung of each preterm lamb. Unbiased sampling involved cutting each left lung into uniformly thick slices, cutting each slice into uniform cubes, and using a random number generator to identify the first cube taken from the top left corner of each slice. Subsequent cubes were taken from every 10th cube across each slice to its bottom right corner (all done by one person). A random number generator was used to select two cubes per slice for embedding and sectioning (done by a second person), followed by image analysis (done by a third person). Image analysis of 10 blocks selected by this unbiased 
sampling method was sufficient to attain $<15 \%$ coefficient of variation for radial alveolar count, volume density of secondary septa, and distal airspace wall thickness for each lung.

\section{Immunoblotting}

Immunoblotting of peripheral, whole-lung homogenate was used to quantify the relative abundance of Cleaved Caspase-3 (Enzo, Farmingdale, NY) and proliferating cell nuclear antigen (PCNA) (BioGenex, Fremont, CA) as previously described by our group (38).

\section{Immunohistochemistry}

We used standard methods to localize cleaved caspase 3 and PCNA in the lung, using the same antibodies that we used for immunoblot (36). Negative immunostaining controls were included (36).

\section{Statistical Analysis}

We used piecewise linear mixed effects regression models to compare the NIS+RN and NIS+ES groups to the NIS group. We modeled the following five outcomes: weight gain, total calories, total fluid, total protein, and total fat. For the weight gain outcome, the two phases of life were defined as postnatal DOL 5-12 and DOL 13-20. Choosing two phases provided a simple model that reflected the biphasic characteristics of the results for the NIS+RN group. The first phase for weight gain began on DOL 5 because preterm lamb weight was unstable during DOL $1-4$, as expected. That is, preterm lamb weight decreased and then increased during postnatal DOL 1-4. For all other outcome variables, the phases were defined as postnatal DOL 1-12 and DOL 13-20. For each outcome, we fit a piecewise linear mixed effect model of the form: Outcome $=$ Group + Phase I + Phase II + Group $^{*}$ Phase I + Group ${ }^{*}$ Phase II + random (Lamb) to account for correlations within the same lmb over time. For each model, we report group differences and 95\% confidence intervals (CIs) in slopes for the first and second phases, and at the final time point (DOL 20).

Simple linear regression models were used to estimate average differences and 95\% CIs for distal airspace wall (DASW) thickness and volume density of secondary septa (Vv sec septa) for the two comparisons: (i) NIS+RN vs. NIS and (ii) NIS+ES vs. NIS. For western blot comparisons, we used nonparametric Mann Whitney $U$-test.

Analyses used R v. 3.2.3 software (R Foundation for Statistical Computing, Vienna, Austria). Statistical significance was assessed at the 0.05 level and all tests were two-tailed.

\section{ACKNOWLEDGMENTS}

We thank the Division of Neonatology at the University of Utah for support.

\section{STATEMENT OF FINANCIAL SUPPORT}

This study was supported in part through a Primary Children's Hospital Foundation Grant, Salt Lake City, Utah (L.A.J-M.), National Institutes of Health (NIH), Bethesda, MD grants K01 DK084036 (L.A.J-M.), R01-HL062875 (K.H.A.), R01 HL110002 (K.H.A.), and T35-HL07744 (K.H.A., Mentor), and the Division of Neonatology, Department of Pediatrics at the University of Utah.

Disclosure: Authors have no conflicts of interest.

\section{REFERENCES}

1. Jobe AH. The new bronchopulmonary dysplasia. Curr Opin Pediatr 2011;23:167-72.

2. Schulzke SM, Pillow JJ. The management of evolving bronchopulmonary dysplasia. Paediatr Respir Rev 2010;11:143-8.

3. Akram Khan M, Kuzma-O'Reilly B, Brodsky NL, Bhandari V. Site-specific characteristics of infants developing bronchopulmonary dysplasia. J Perinatol 2006;26:428-35.

4. Bhandari V. Drug therapy trials for the prevention of bronchopulmonary dysplasia: current and future targets. Front Pediatr 2014;2:76.

5. Jobe AH. What is BPD in 2012 and what will BPD become? Early Hum Dev 2012;88 Suppl 2:S27-8.

6. Reyburn B, Li M, Metcalfe DB, et al. Nasal ventilation alters mesenchymal cell turnover and improves alveolarization in preterm lambs. Am J Respir Crit Care Med 2008;178:407-18.

7. Null DM, Alvord J, Leavitt W, et al. High-frequency nasal ventilation for 21 $\mathrm{d}$ maintains gas exchange with lower respiratory pressures and promotes alveolarization in preterm lambs. Pediatr Res 2014;75:507-16.
8. Coalson JJ. Pathology of bronchopulmonary dysplasia. Semin Perinatol 2006;30:179-84.

9. Albertine KH. Utility of large-animal models of BPD: chronically ventilated preterm lambs. Am J Physiol Lung Cell Mol Physiol 2015;308: L983-L1001.

10. Stephens BE, Walden RV, Gargus RA, et al. First-week protein and energy intakes are associated with 18-month developmental outcomes in extremely low birth weight infants. Pediatrics 2009;123:1337-43.

11. Biniwale MA, Ehrenkranz RA. The role of nutrition in the prevention and management of bronchopulmonary dysplasia. Semin Perinatol 2006;30:200-8.

12. Frank L, Sosenko IR. Undernutrition as a major contributing factor in the pathogenesis of bronchopulmonary dysplasia. Am Rev Respir Dis $1988 ; 138: 725-9$.

13. Fanaro S. Feeding intolerance in the preterm infant. Early Hum Dev 2013;89 Suppl 2:S13-20.

14. Massaro D, Massaro GD, Baras A, Hoffman EP, Clerch LB. Calorie-related rapid onset of alveolar loss, regeneration, and changes in mouse lung gene expression. Am J Physiol Lung Cell Mol Physiol 2004;286:L896-906.

15. Rehan VK, Sakurai R, Li Y, et al. Effects of maternal food restriction on offspring lung extracellular matrix deposition and long term pulmonary function in an experimental rat model. Pediatr Pulmonol 2012;47:162-71.

16. Marx CM, Smith PG, Lowrie LH, et al. Optimal sedation of mechanically ventilated pediatric critical care patients. Crit Care Med 1994;22:163-70.

17. McPherson C. Sedation and analgesia in mechanically ventilated preterm neonates: continue standard of care or experiment? J Pediatr Pharmacol Ther 2012;17:351-64.

18. Doherty TJ, Andrews FM, Provenza MK, Frazier DL. The effect of sedation on gastric emptying of a liquid marker in ponies. Vet Surg 1999;28:375-9.

19. Lydon AM, Cooke T, Duggan F, Shorten GD. Delayed postoperative gastric emptying following intrathecal morphine and intrathecal bupivacaine. Can J Anaesth 1999;46:544-9.

20. Colombato LA, Sabbà C, Polio J, Groszmann RJ. Influence of anesthesia, postanesthetic state, and restraint on superior mesenteric arterial flow in normal rats. Am J Physiol 1991;260(1 Pt 1):G1-6.

21. Gumbleton M, Nicholls PJ, Taylor G. Differential influence of laboratory anaesthetic regimens upon renal and hepatosplanchnic haemodynamics in the rat. J Pharm Pharmacol 1990;42:693-7.

22. Hay WW Jr. Strategies for feeding the preterm infant. Neonatology 2008;94:245-54.

23. Massaro D, Alexander E, Reiland K, Hoffman EP, Massaro GD, Clerch LB. Rapid onset of gene expression in lung, supportive of formation of alveolar septa, induced by refeeding mice after calorie restriction. Am J Physiol Lung Cell Mol Physiol 2007;292:L1313-26.

24. Joss-Moore L, Carroll T, Yang Y, et al. Intrauterine growth restriction transiently delays alveolar formation and disrupts retinoic acid receptor expression in the lung of female rat pups. Pediatr Res 2013;73:612-20.

25. Ramel SE, Brown LD, Georgieff MK. The impact of neonatal illness on nutritional requirements-one size does not fit all. Curr Pediatr Rep 2014;2:248-54.

26. Ehrenkranz RA, Das A, Wrage LA, et al.; Eunice Kennedy Shriver National Institute of Child Health and Human Development Neonatal Research Network. Early nutrition mediates the influence of severity of illness on extremely LBW infants. Pediatr Res 2011;69:522-9.

27. Ehrenkranz RA. Early, aggressive nutritional management for very low birth weight infants: what is the evidence? Semin Perinatol 2007;31: 48-55.

28. deRegnier RA, Guilbert TW, Mills MM, Georgieff MK. Growth failure and altered body composition are established by one month of age in infants with bronchopulmonary dysplasia. J Nutr 1996;126:168-75.

29. Wemhöner A, Ortner D, Tschirch E, Strasak A, Rüdiger M. Nutrition of preterm infants in relation to bronchopulmonary dysplasia. BMC Pulm Med 2011;11:7.

30. Bhandari V, Bergqvist LL, Kronsberg SS, Barton BA, Anand KJ; NEOPAIN Trial Investigators Group. Morphine administration and shortterm pulmonary outcomes among ventilated preterm infants. Pediatrics 2005;116:352-9. 


\section{Articles | Joss-Moore et al.}

31. Kim SH, Li M, Pyeon TH, So KY, Kwak SH. The volatile anesthetic sevoflurane attenuates ventilator-induced lung injury through inhibition of ERK1/2 and Akt signal transduction. Korean J Anesthesiol 2015;68:62-9.

32. Romero A, Moreno A, García J, Sánchez C, Santos M, García J. Effects of sevoflurane on ventilator induced lung injury in a healthy lung experimental model. Rev Esp Anestesiol Reanim 2016;63:22-8.

33. Frank L, Groseclose E. Oxygen toxicity in newborn rats: the adverse effects of undernutrition. J Appl Physiol Respir Environ Exerc Physiol 1982;53:1248-55.

34. Martin CR, Dasilva DA, Cluette-Brown JE, et al. Decreased postnatal docosahexaenoic and arachidonic acid blood levels in premature infants are associated with neonatal morbidities. J Pediatr 2011;159:743-749.e1-2.
35. Joss-Moore LA, Wang Y, Baack ML, et al. IUGR decreases PPAR $\gamma$ and SETD8 Expression in neonatal rat lung and these effects are ameliorated by maternal DHA supplementation. Early Hum Dev 2010;86:785-91.

36. Albertine KH, Dahl MJ, Gonzales LW, et al. Chronic lung disease in preterm lambs: effect of daily vitamin A treatment on alveolarization. Am J Physiol Lung Cell Mol Physiol 2010;299:L59-72.

37. Rehan VK, Torday JS. PPAR $\gamma$ signaling mediates the evolution, development, homeostasis, and repair of the lung. PPAR Res 2012;2012:289867.

38. MacRitchie AN, Albertine KH, Sun J, et al. Reduced endothelial nitric oxide synthase in lungs of chronically ventilated preterm lambs. Am J Physiol Lung Cell Mol Physiol 2001;281:L1011-20. 